\title{
AUTOMATED ICT SYSTEMS IN INLAND WATERWAYS BY DEVELOPING A MULTI- FLOW RIVER INFORMATION SERVICES SYSTEM
}

\author{
K.J. James
}

Associate Professor, (Rtd.), Department of Ship Technology, Cochin University of Science and Technology, Cochin, India

Vikas V. Shenoy

Managing Director, Padmasree Developers, Cochin, India

\section{Bhasi}

Professor, School of Management Studies, Cochin University of Science and Technology, Cochin, India

\section{G. Nandakumar}

Professor (Rtd.) Department of Ship Technology, Cochin University of Science and Technology, Cochin, India

\begin{abstract}
The objective of any River Information Services system is the safe, efficient and environment friendly transport of men, materials and machinery in inland waterways, including canals, lakes and backwaters. Transport should be safe with minimum injuries, fatalities and accidents. It should efficiently maximise throughput and effective capacity of waterways and carrying capacity of vessels, reduce travel time and costs and should efficiently link between transport modes and terminals. Environment hazards like polluting emissions and oil spills due to illegal actions or normal operations should be avoided.

The RIS can be categorised into various information levels like fairway information, tactical and strategic traffic information, traffic and transport logistics management information, statistical information, calamity abatement information, tariff information and information for law enforcement. The various services and their functions are interlinked with multiple users and various levels of operation. Because of this, information flow is multilevel for multiple users. In RIS systems, the RIS personnel evaluate the various data and make decisions based on personal experiences and similar business situations and then disseminate information to multiple users. Human errors in such a situation can lead to accidents with catastrophic effects like loss of business, loss of vessels and even loss of lives. In such situations computerised information processing systems can play a significant role in improving safety and
\end{abstract}


security in Inland Water Transport (IWT). Here the human element is replaced by Automated River Information Services (ARIS) system. Since the information flow in RIS systems is multilevel and there are multiple users, complexity of information processing and dissemination is of a higher order. As an improvement on existing RIS systems, this paper proposes the development of Automated River Information Services system.

It is an interactive software based system intended to make decisions compiling useful information from raw data, documents and business models to identify and solve problems. In this system, human intervention is substituted by an Artificial Intelligence system which is fully automated, leading to better efficiency and security of operation. ARIS architecture has been developed and described in this paper. This software will prove useful in the IWT sector, especially when many countries are moving towards eco-friendly means of transport, according higher priority for Inland Water Transportation.

Keywords: River information services system; strategic, dynamic and urgent information; multilevel and multi flow information, Automated river information services system

Cite this Article: K.J. James, Vikas V. Shenoy, M. Bhasi, C.G. Nandakumar, Automated ICT Systems in Inland Waterways by Developing a Multi-flow River Information Services System, International Journal of Advanced Research in Engineering and Technology, 10 (2), 2019, pp 389-402.

http://iaeme.com/Home/issue/IJARET?Volume=10\&Issue $=2$

\section{INTRODUCTION}

River Information Services (RIS) can be defined as "the harmonised information services being provided with the objective of supporting traffic and transport management in inland waterways including canals, lakes and backwaters, enhancing safety, security, environmental friendliness and the quality \& efficiency of transport services"(1). RIS systems depend on input data and information flow among various stakeholders like waterway authorities, skippers (personnel manning the vessels), RIS operators, lock and bridge operators, terminal operators, calamity centre personnel, fleet managers, cargo shippers, consigners, consignees, freight brokers and supply forwarders. RIS systems are designed for enhancing the safety, security and efficiency of Inland Water Transport (IWT) operations. Protection of environment is a major consideration in designing RIS systems.

River transport is not a standalone concept and it has to be part of a transport chain including other modes like roads, railways and sea $\&$ coastal transport, which together form a multimodal transport system. Inland waterways include rivers, canals, lakes and even ports in river mouths. RIS systems consist of one or more harmonized information \& communication technology (ICT)systems intended to process information about inland water transport. For this, human resources, hardware, software, communication means etc. have to be provided at the RIS center. Command area under an RIS system may include one or more countries since in many instances rivers flow through different countries and they form the boundary between countries. Compared to road traffic, accidents are rare in IWT sector, primarily because the number of vessels plying in the rivers is limited. When more and more vessels start moving in the waterways, escalation of accident rates is bound to occur, primarily due to human error. "Accidents do not happen, they are caused due to negligence"- this is the basic concept behind any safety programme and safety system.

The various services and their functions are interlinked with multiple users and various levels of information. Because of this, information flow will be multilevel for multiple users. 
Automated ICT Systems in Inland Waterways by Developing a Multi-flow River Information Services System

RIS systems are handled by the operating personnel who compile useful information from raw data, documents, personal knowledge and business models to identify and solve problems. Human errors in such situations can be disastrous and costly and even loss of lives can occur. Environmental issues like pollution because of oil spills, faulty emissions etc. can also have detrimental effects on environment. In such situations the relevance of Automated RIS becomes evident wherein the human element is replaced by a computerised information processing system which may be named as Automated River Information Services (ARIS) system.

"Modern logistics management requires intensive information exchange between partners in logistics chain. RIS systems facilitate inland water transport organisation and management. Through information exchange, transport operation(such as trip schedules and terminal/lock operation plans) can easily be optimised, providing advantages for inland navigation and enabling it to be integrated into the intermodal logistics chains"(2).

\section{INFORMATION FLOW}

\subsection{Functional decomposition of RIS}

Information can be categorised as static, dynamic and urgent information. Information regarding navigational aids and traffic signs, physical limitations in the fairway like bridges, locks etc. and navigational rules \& regulations are of static nature. Static information has to be communicated on a scheduled regular basis. Actual meteorological information, present and probable future water levels based on tidal/weather information at gauges etc., are dynamic in nature and are time-dependant. Dynamic information has to be communicated on a real-time basis and require frequent updating. Temporary obstructions in the fairway, accidents/incidents in the fairway, coordination of assistance of patrol vessels etc. are urgent in nature. Urgent information has to be communicated immediately to the concerned parties and authorities.

Functional decomposition of RIS enables the allocation of information supply in response to user demand. Proper access, assessment, processing and dissemination of information to various users are multilevel and the process is really complex, since there are multiple stakeholders. Same function may serve several participants in the transport process. For example, geography of the navigational area and its updates have to be communicated to the vessel master, vessel traffic services(VTS) operator, lock/bridge operator, waterways authority, terminal operator, calamity centre, fleet manager and cargo shipper. Thus, it is evident that the flow of information is multilevel and it has to be forwarded to multiple stakeholders.

\subsubsection{Fairway information service}

Fairway information consists of information regarding the geography of the navigation area, water depth contours in the navigation fairway, long term and temporary obstructions in the fairway, navigational aids and traffic signs, meteorological information, restrictions caused by flood \&ice, land slide \& rock fill, physical constraints like locks / bridges and their operating times, navigational rules, regulations $\&$ recommendations and rates of waterway infrastructure charges. Among these, the ones regarding temporary obstructions, restrictions caused by flood\& ice, landslide \& rock-fill and malfunctions of aids to navigation fall under urgent category. Real time meteorological information, present and future water levels at gauges and state of rivers and canals, locks and bridges in the RIS are of dynamic nature in the sense that these are time dependant. All other fairway information can be grouped under static category.

In comparison to traditional communication systems like radio, telephone etc. recent developments like smart phones provide smarter options for disseminating fairway information. As much as possible, fairway information services should be provided as tailor-made through approved communication modes by a competent authority only. Vessel to vessel and vessel to shore services should deal exclusively with the safety of personnel and safety of traffic of 
vessels. Standard vocabulary should be followed for notice to skippers because automatic translation to other languages will be required especially when vessels cross boundaries of different countries with different languages. Electronic navigation charts (ENC) presenting fairway information should be prepared observing electronic chart display and information system(ECDIS) standards for information mode.

\subsubsection{Traffic information}

Traffic information consists of tactical and strategic information. Presentation of own vessel's position and presentation of other vessel's positions are of dynamic nature. They are short term related and require frequent updating. Tactical traffic information makes use of radar as well as vessel tracking and tracing systems such as automatic identification systems (AIS). Strategic traffic information makes use of electronic vessel reporting systems like data base of vessel's cargo, voice and data by VHF etc. Strategic traffic information which are medium and long term related include presentation of fairway information, presentation of vessel characteristics and presentation of intended destinations which are static in nature. Presentation of vessel's position in large surroundings, medium and long term assessment of traffic situation, presentation of cargo characteristics and organization \& regulation of traffic flow are categorised as dynamic. Presentation of information on accidents/incidents in the coverage area is of urgent nature and requires immediate action.

\subsubsection{Traffic management}

Traffic management is done by vessel traffic services (VTS) centre and emphasis is given to traffic organization considering the local difficult situations such as narrow fairways, bends, narrow bridges, faster water currents etc. Presentation of vessel's position in large surroundings, monitoring of passing and manoeuvring arrangement, short term assessment of traffic situations and organisation \& regulation of traffic flow in RIS coverage area are of dynamic nature and has to be updated real -time.

Navigation support consists of nautical support and vessel services support. Nautical support is provided by tug boats and mooring personnel. Bunker boats, waste oil removal boats, vessel equipment firms and repair organizations provide vessel services support. Information to pilots, information to tug boats, information to bunker boats etc. are dynamic in nature and come under navigational support.

\subsubsection{Calamity abatement support}

At the beginning of the voyage, the vessel has to be registered at the RIS centre. Transport data are uploaded and updated during the voyage. In case of accidents, details will be communicated to the emergency services without delay. The skipper (vessel master) shall provide the required data. The vessel should be provided with appropriate means of communication. The vessel's position and data should be reported while entering and leaving the RIS area and also at specified reporting points within the RIS area. Any change in data should be reported. If there are any stoppages for longer periods, report should be sent at the commencement and end of the stoppage period. This information is of urgent nature.

\subsubsection{Information for transport logistics}

Transport logistics management consists of transport management, intermodal port \&terminal management and cargo \& freight management. Transport management deals with information about provision and presentation of estimated time of arrival (ETA) of vessels, voyage plans, information on free loading space, performance of contracted transports and terminals, unusual threats like strikes, fall in water levels, matching transport at terminal performance with service levels agreed upon and adjustments to methods for voyage plan. Inter modal port and terminal management deals with actual terminal/port status information on vessels being loaded and unloaded and required time of arrival (RTA) of these vessels, waiting places and positions. 
These are dynamic in nature and will be communicated to the terminal operator, cargo shipper and vessel master. Planning requires information on ETA of approaching vessels, medium and long term schedule of terminal processes and medium and long term RTA of vessels. These are static information to be communicated to terminal operators. Cargo and fleet management requires information on fleet of vessels and their transport characteristics and also information on the cargo to be transported. These are dynamic in nature and will be communicated to the fleet manager and cargo shipper.

Voyage planning is the task of the skipper and the vessel owner .Transport management is done by freight brokers and transport service quality managers. Port and terminal management falls under the purview of the vessel masters, terminal operators and cargo shippers.

\subsubsection{Information for law enforcement}

Law enforcement ensures that people within a given jurisdiction adhere to the laws of that jurisdiction. Vessel operators should stick to the laws of the land when national boundaries have to be crossed and immigration, customs clearance etc. are mandatory. Traffic safety rules and environmental regulations also have to be attended to. Information for law enforcement (ILE) deals with cross border management, immigration of personnel, traffic safety regulations and environment protection rule This information are of static nature.

\subsubsection{Statistical information}

This type of information regarding freight statistics is generally required for monitoring, bench marking and future planning. Waterway authorities should note and record the transit of vessels, type of vessels, capacity, cargo etc. at certain points (locks, ports etc.) of the waterway in such a way as to conduct future statistical analyses. Electronic data collection will facilitate the process for data providers and statistical offices. These statistics are of interest to the waterway authorities, international organisations and companies engaged in inland navigation for strategic planning and monitoring. These data are time dependant and hence dynamic in nature

\subsubsection{Waterway charges and harbour dues}

Waterway charges and harbour dues which are normally static in nature are also informed through RIS centre. If there are changes in the waterway charges, communication has to be sent to the affected parties. Pending arrears, if any, may be brought to the notice of the vessel owner in advance. The main users are vessel masters, lock\& bridge operators, waterway authorities and cargo shippers. The travel data of the ship can be used to automatically calculate the charges and initiate invoicing, thus facilitating the process for waterway users and authorities.

\subsection{Characteristics of information flow}

In the RIS system, data will be provided by the service providers and skippers. These data will be analysed by the RIS centre personnel who evaluate, asses and process data and decide upon the course of action. Then appropriate information has to be communicated to the right user at the right time and the right place. At this juncture, human element comes into play. Information provided can have three main deficiencies.

(a) Errors can creep in while communicating the information rendering the message invalid and useless.

(b) Delay can occur in communicating the plan of action or any other information which can prove costly/ damaging to the IWT operator.

(c) Insufficient or incomplete information can also hinder the decision making.

All these can lead to the breakdown of the intended objectives/functions of RIS and prove as hindrances in the smooth functioning of the IWT sector. 
RIS supports a number of management tasks in inland shipping. These tasks are related to the objectives and performed in three different arenas:-

(a)Transport logistics where parties that organise the cargo cooperate with the parties that organise transport.

(b) Transport where parties that organise the transport cooperate with the parties that execute the transport

(c)Traffic where parties that execute the transport cooperate with those managing the resulting vessel traffic.

For each individual task an information processing loop may be drawn. This can be used to define the RIS functions. The efficiency of execution of a task can be enhanced by using one or more services. Thus it is evident that the information flow is multi-level and complicated and there is ample scope for automation in the river information flow.

No international definition of automation levels in inland navigation is currently available. The Central Commission for Navigation on the Rhine (CCNR) who are the pioneers in utilising IWT to its fullest extent have in its "Vision 2018 Document" set itself a number of ambitious objectives for the next five years which will contribute to the sustainable development in inland navigation in ecological, social and economic terms. It states that "Quality information services, making use of innovative technologies will be used by the shipping industry in order to both improve the safety of inland navigation and take more account of logistics aspects and ecological concerns"(3). In January 2018, CCNR proposed definitions of levels of automation in inland navigation. Technological developments in automated navigation like drones and autonomous road vehicles have prompted inland navigation to be more interested in automation, from simple navigational assistance to fully automated navigation.

As suggested in theabove mentioned vision document, this paper proposes the development of information flow architecture for an Automated RIS

\section{AUTOMATED RIVER INFORMATION SERVICE (ARIS)}

ARIS are a specific class of information systems that support organisational decision making activities in the IWT business. In a conventional RIS centre, the personnel at the centre compile various data and identify the problems. They have to solve the problems and make decisions for implementing the solution. In this decision making process errors can creep in, causing severe damage in the IWT sector. In such a situation, development of an Automated RIS becomes relevant. A properly designed ARIS is an interactive software based system intended to make decisions, compiling useful information from raw data, documents and business models to identify and solve problems. Providing technically feasible and financially viable practical solutions to management problems in IWT is the basic objective of developing ARIS.

The main stakeholders interested in developing ARIS are the policy makers, regional managers, system engineers, service providers and various users. Owners of the vessels and river authorities are concerned with the safety of transport and they are the policy makers. The objectives, tasks and the required services are dictated by the policy makers. They discuss the functions and information needs for providing services along with operating constraints. Interest of the vessel owners will be in providing transport and logistical information to the shipper of cargo and also the terminal operators.

Regional managers such as traffic control managers, waterway authorities (managers), managers of search and rescue operations, vessel owners and cargo shippers define their requirements. They provide accurate description of the services, local aspects and man/machine interface. System engineers integrate the hardware and software components and prepare the system specifications. System integrators, telecom operators and RIS and vessel tracking 
system (VTS) suppliers undertake this task. The system components are combined into complete systems for providing ARIS. Service providers develop, maintain and operate the ARIS applications aided by the users who provide the main input to the application themselves and also control the autonomous applications.

The beneficiaries of the ARIS are skippers(vessel masters), RIS operators, lock and bridge operators, waterway authorities, terminal operators, calamity center personnel, fleet managers, cargo shippers, consigners, consignees, freight brokers and supply providers. They also happen to be the people who provide the data which is the main input for the system.

\section{4. 'ARIS' ARCHITECTURE}

In a river information services system, the data providers and users of information are practically the same group of people like vessel masters, VTS operators, waterway authorities, terminal operators etc. The data provided by one group becomes information for other groups. In some situations the data providers themselves become the users. The various services and their functions are interlinked with multiple users and different levels of information. When a data provided by the service provider is processed according to the rules and regulations set by the policy makers and validated by the regional managers, it becomes information for the users. The users of this information are the very same group that provides the data and hence it is a closed loop system.

The data provided by the vessel master to the RIS centre regarding estimated time of arrival (ETA) becomes information for other groups like terminal operator, cargo shipper, supply forwarder, vessels supply organisations like bunker boats, repair teams and transport service quality managers. If the vessel has to pass through locks, the lock operator also has to be informed. If an accident occurs in the waterway, vessel master will inform the RIS centre. The centre will immediately inform the calamity centre personnel, who are responsible for calamity abatement activities. The calamity centre informs fire \& rescue team, patrol vessels, police team, etc. for rescue operations. If the vessel has to be towed to a safe position, towing vessels also have to be informed

Depending upon the criticality of the data, the ARIS centre will have to identify the groups to which information has to be sent immediately. So also ARIS identifies the groups for which the information will be useful and relevant; and it will be sent to those groups as well. Hence the information processing and forwarding is a complex process; it has to be forwarded to multiple users and the flow will be multilevel.

The various levels in the ARIS architecture can be grouped into policy makers, regional managers, system engineers and users \& service providers.

\subsection{Policy makers}

Waterway authorities and organisation of vessel owners have their own policy objectives, tasks and ideas regarding the services required to achieve the objectives. The prime requirement of policy makers is to solve the traffic and transport related problems. They dictate policies and set rules and regulations which have to be validated by the regional managers. The policy makers want a safe and secure traffic and transport for which they define the objectives. Once the objectives are defined, the various tasks to be fulfilled are determined. Waterway authorities, law enforcement authorities, calamity centre personnel etc. belong to this category. Another category of policy makers are the organisation of ship owners and fleet operators. The policy makers dictate the services required to achieve their objectives. Once the required services are defined, various functions and the information required for accomplishing these functions have to be determined. These functional requirements will have to satisfy the 
boundary conditions which may be certain restrictions during the interaction with other functions and information needs. For example, when rivers flow through different countries or states, where the rules and regulations are different and local conditions also differ, they have to be modified according to the local conditions. Thus localisation and validation of the rules and regulations have to be done by the regional managers.

\subsection{Regional managers}

Waterway managers, managers of search and rescue operations, vessel owners and cargo shippers fall into the category of regional managers. They control the RIS applications. They are the ones who define the requirements for applications. They are more familiar with the local aspects and conditions and hence can give accurate descriptions of the services and functions. Waterway managers, traffic control managers, managers of search and rescue operations, ship owners and cargo shippers come under the category of regional managers. They define the requirements to the system engineers and also do the validation and localisation of the design systems.

\subsection{System engineers}

System engineers prepare system components which are combined to enable ARIS services. RIS\&VTS suppliers, system integrators and telecom operators combine the system components and develop the programme for information processing and enable the ARIS. Thus ARIS will control autonomous applications and enable the services. They prepare the system specifications and integrate hardware and software components into system components.

\subsection{Service providers and users}

Service providers along with the users feed data to the ARIS. Appropriate data is chosen, processed and automatically forwarded to the targeted user for taking necessary precaution and monitoring followed by corrective action. With proper programming and coding, ARIS can be designed to handle all possible scenarios and provide appropriate information to the user thereby minimising the error that would have crept in, had this been handled by the operating personnel. Thus ARIS provides reliable and relevant information for the users.

Under the service providers category the following groups may be listed:-VTS operators, Lock and bridge operators, Waterway authorities, Terminal operators and Calamity centre personnel

Under the users category the following personnel may be listed:- Vessel masters, Fleet managers, Cargo shippers, Vessel supplies organisers, Bunker suppliers, Repair parties, Freight brokers and Transport service quality managers 
Automated ICT Systems in Inland Waterways by Developing a Multi-flow River Information Services System

ARIS Architecture- Flow Chart

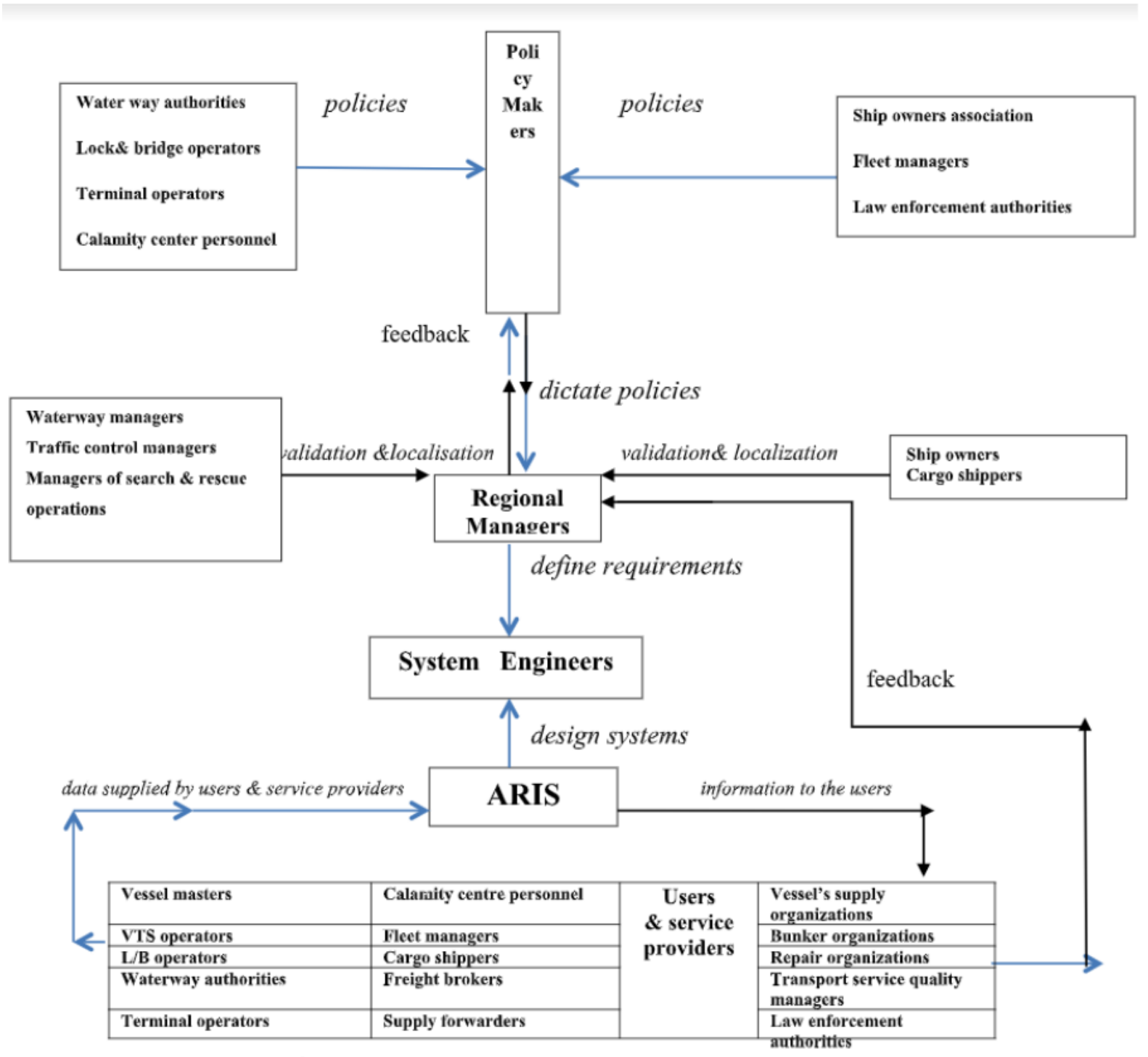

Figure 1 ARIS architecture- flow chart

\section{FUNCTIONAL OBJECTIVES OF ARIS}

The objective of any RIS system is the safe, efficient and environment friendly transport of men materials and machinery in inland waterways. These are the goals to be achieved by any efficient RIS system."The three main objectives may be stated as safety, efficiency and environment friendliness" ${ }^{\text {(4). }}$.

\subsection{Functions of ARIS}

The functions of the ARIS may be classified as information flow, traffic control, vessel health certification, operational health certification and green health certification.

\subsubsection{Information flow}

In the inland water transport sector, there is a lot of complex information flow among multiple service providers and users. This leads to complicated information networking systems. When this is handled manually, there will be ambiguity and possibility of human error. Hence this paper suggests a model of developing an ARIS system to handle complex information systems 
by collecting data from various service providers and users and processing it to provide information to multiple agencies depending upon the traffic situation.

\subsubsection{Traffic control}

Information regarding the traffic situation may be provided as tactical traffic information and strategic traffic information. Tactical traffic information are short term related and decisions have to be of urgent nature. Presentation of own vessels position and presentation of other vessels position relative to own vessel have to be communicated among the ship master, VTS operator, and lock/ bridge operator so that traffic decisions can be taken on an urgent basis. Strategic traffic information are medium and long term related. Presentation of fairway information, vessels position in large surroundings, medium and long term assessment of traffic situation, vessels characteristics, cargo characteristics, intended destination, information on incidents/ accidents in the coverage area and organisation \& regulation of traffic flow are to be communicated among various stake holders. ARIS can perform the traffic control function very effectively without human intervention.

\subsubsection{Vessel health certification}

Certification regarding the seaworthiness of the vessel is mandatory in all countries. "Vessels have to be surveyed before they are put into service. Also periodical survey once in every twelve months is mandatory. Additional surveys as occasions demand have also to be done. Drydocking has to be conducted every twenty-four months"(5). Based on the dimensions of the vessel and the power of the propulsion system, vessels will be categorised as class A and class $\mathrm{B}$ vessels. Renewal of certification also has to be done periodically to assess the safety and seaworthiness of the vessels. These data have to be communicated to the ARIS centre while registering with the ARIS.

\subsubsection{Operational health certification}

As per the Inland Vessels Certification Rules applicable to the particular region, vessels have to be operated by certified personnel only. Certificate of competency and certificate of service have to be issued by the statutory authorities. Operation of the vessel by uncertified, inexperienced and incompetent personnel will lead to accidents and chaos in the IWT sector. This has to be avoided at any cost. Depending upon the class of the vessel and the carrying capacity, the vessels should have appropriately certified operating personnel on board. Age, qualification and certification of the operating crew like first-class master's certificate, second class master's certificate, serang`s certificate, lasker`s certificate, first class engine driver's certificate, second class engine driver's certificate and serang-cum-driver's certificate will be prescribed by the competent authority. ARIS can ascertain that the operating personnel are having appropriate qualifications and certification and that the vessel can operate smoothly in its area of control. Thus the operational health of the vessel may be ascertained with the help of ARIS. Thus the operational health of vessels can be monitored regularly

\subsubsection{Green health certification}

Air pollution due to IWT had not been of much concern in the last decades. However "the undisputed competitive position of IWT in the field of emissions when compared with road transport is increasingly contested. The gap regarding emissions to air between road transport and IWT is rapidly becoming smaller. In contrast to road haulage sector, the emission standards for new engines are much less stringent and the average life time of engines in inland vessels is very long"(6). When more and more vessels start operating in the inland waterways, pollution problems are bound to increase. ARIS aids in continuous and regular monitoring of pollution norms. By enforcing stricter norms the emissions from the engines can be reduced. .ARIS also provides for continuous monitoring while carrying dangerous cargoes. 
Automated ICT Systems in Inland Waterways by Developing a Multi-flow River Information Services System

\subsection{Advantages of ARIS}

The main beneficiaries of ARIS are the service providers and users. Benefits accruing from implementation of ARIS for service providers and users may be stated as follows:

\subsubsection{Service providers.}

\subsubsection{VTS operators}

VTS operators are the key personnel in ensuring the safety of navigation in difficult local situations and providing protection for the surrounding human population and infrastructure from potential dangers of shipping. ARIS provides for improved safety and protection by reducing the element of human intervention in the decision making process. In situations like narrow fairways and shoals, narrow bends, narrow and many bridges, faster water currents and cross currents, conjunctions of fairways and fairways with traffic regulations, ARIS plays a prominent and innovative role. This is especially true in a futuristic situation where a higher density of inland water transport is anticipated.

\subsubsection{Lock and bridge operators}

Lock and bridge planning is to be supported with a vessel tracking and tracing system with a data base and suitable means of communication. The ARIS can optimise the traffic flow by supporting the lock and bridge management in short term decisions for planning of the lock and bridge cycle by presentation of an electronic lock diary, by a database and by registration of waiting times. By exchanging data with neighbouring locks and transmission of waiting times to the shippers, the lock operator and skipper are benefited. Also by calculating the ETA and Required Time of Arrival (RTA) of various vessels for a chain of locks and transmitting RTA to the shippers, the operation of locks and bridges can be optimised.

\subsubsection{Waterway authorities}

Waterway authorities should be provided with details regarding fairway information, strategic traffic information, information on medium and long term schedule of lock and bridge operations, calamity abatement support and also information for law enforcement, statistics, and waterway charges \& harbour dues. ARIS improves the efficiency of operation by automation of the data processing and information services.

\subsubsection{Terminal operators}

The terminal operators should be provided with data regarding water depth contours in the navigational channel, details of vessels characteristics and cargo details, intended destination etc. For port and terminal planning ETA of approaching vessels, medium and long term schedule of terminal processes and medium and long term RTA of vessels have to be provided by the ARIS. Also intermodal port and terminal management, presentation of vessels waiting, vessels being loaded and unloaded, actual status of terminal process, RTA of vessels, waiting places and positions have to be forwarded to the ARIS. All these interactive functions can be successfully accomplished by employing ARIS.

\subsubsection{Calamity centre personnel}

These personnel are the ones to provide calamity abatement support. Accidents usually occur because of human error. Information on incidents focussed on traffic situation, assessment of traffic situation in such cases, coordinating assistance of patrol vessels etc. have to be done by the ARIS. Assessment of possible effects of the accident on environment, people and traffic, presentation of information to patrol vessels, police boats and fire squad boats are done in a more efficient manner with the implementation of ARIS. Initiation and coordination of search and rescue operations, taking measures on traffic control and environmental and people protection can also be better accomplished with the implementation of ARIS. Once the calamity is over and no further action is required, all the contacted people have to be informed. 


\subsubsection{Users of ARIS}

\subsubsection{Vessel masters}

Tactical and strategic traffic information like vessels position, traffic situation, vessels characteristics and cargo characteristics have to be communicated to the ARIS centre by the vessel master.

Fairway information like geography of the navigational area and its updates, water depth in the navigation channel, navigation aids and traffic signs, bridges, temporary and long term obstructions in the fairway, water levels at gauges, meteorological information, flooding \& ice, lock/bridge operating timings, navigation rules and regulations and rates of waterway infrastructure charges have to be communicated to the vessel master by ARIS. Navigational support, lock and bridge operation ,medium and long term RTA of vessels, information on incidents focussed on traffic situation, initiation and coordination of search and rescue operations are organised ina more efficient manner with the implementation of ARIS. Information for transport management and transport logistics also are in the purview of ARIS. Presentation of RTA of vessels, waiting places and positions, medium and long term RTA of vessels etc. are to be provided by the ARIS. Again, waterway charges and harbour dues have to be calculated and communicated to the vessel master. All these activities can be efficiently organised with ARIS thereby improving the overall performance of IWT.

\subsubsection{Fleet managers}

Fleet managers are to be provided with fairway information like geography of the navigational area and its updates, water depth contours in the navigational channel, present and probable water levels at gauges, restrictions caused by ice \& floods, regular lock/bridge operating times, physical limitations like locks \& bridges, navigational rules $\&$ regulations and rates of waterway infrastructure charges. Also information to auxiliary service providers like bunker boats, water barges waste oil removal boats etc. can be better serviced by ARIS. Synchronisation of these activities will be better accomplished by the ARIS.

Traffic information like presentation of vessels positions in large surroundings, characteristics of the vessel and the cargo characteristics are to be provided by the fleet manager to the ARIS. Information for transport logistics like voyage planning, information on fleet of vessels and their transport characteristics and details regarding the cargo to be transported have to be communicated to the ARIS.

Thus ARIS will improve the performance of the system and will be of great help to the fleet managers.

\subsubsection{Cargo shippers}

Cargo shippers are the ones who generate revenue for the IWT operations. They have to be informed regarding fairway geography of the navigational area, water depths, restrictions in the waterways, lock and bridge operating timings, rates of infrastructure charges etc., by ARIS

Information on intended destination, RTA at final destination, type of cargo, details for transport logistics, transport management, intermodal port and terminal management, cargo fleet management and details regarding vessel characteristics and cargo characteristics have to be informed to the ARIS by the cargo shipper. All these functions can be accomplished faster and more efficiently with ARIS

\subsubsection{Vessel supplies organisations}

Supply vessels like bunker barges and water barges are an integral part of the IWT process. Required information regarding ETA and RTA of vessels have to be provided to these organisations so that they will be in place at the right time. ARIS can provide accurate information to these organisations ina fast and more efficient manner. 
Automated ICT Systems in Inland Waterways by Developing a Multi-flow River Information Services System

\subsubsection{Bunker suppliers}

Sufficient bunkers have to be carried on board for the proposed voyage. Carrying more fuel will reduce payload. Information regarding the required quantity and the location at which it is available has to be informed to the shipmaster and fleet manager. This service becomes more efficient with the implementation of ARIS.

\subsubsection{Repair parties}

Docking for repairs entails opportunity costs in addition to repair charges. If some of the repairs could be done during voyage, it will be advantageous. Planning for such repairs is better coordinated with ARIS.

\subsubsection{Freight brokers}

Freight brokers are the market makers for the entire IWT operations. They know where the cargo is available and where the markets are. This database forms the backbone of the whole trade. Information provided by the ARIS will be greatly useful for these people and organisation of cargo can be done more efficiently.

\subsubsection{Transport service quality managers}

These people do the transport management by controlling overall performance of the contracted fleet managers and skippers and terminal operators. They control the progress of the contracted transports. Monitoring unexpected threats for the reliability of these transports and finalising these transports (invoicing and delivery) are the tasks of these managers. ARIS will definitely improve the performance of these people by providing them with right information at the right time.

\section{CONCLUSIONS}

In the inland water transport sector, there is a lot of complex information flow among multiple service providers and users. This leads to complicated information networking systems. When this is handled manually, there will be ambiguity and possibility of human error. This paper suggests a model of developing an ARIS system to handle complex information systems by collecting data from various service providers and users and processing it to provide information to multiple agencies depending upon the traffic situation. The functions of ARIS are defined as information flow, traffic control, vessel health certification, operational health certification and green health certification.

The entire operation of collecting static, dynamic and urgent information from various sources, processing and communicating the required output at the appropriate time to multiple users is a complex process. It is observed that information processing using AI systems increases the efficiency of the entire operations. The proposed ARIS is developed by modifying the existing RIS systems.

ARIS architecture has been proposed defining logical information flow among various stake holders. The policy makers and regional managers have been identified for the development of the rules of the ARIS and their duties and responsibilities have been defined. Benefits of implementing ARIS for the various stakeholders have been enumerated. The software developed will prove useful in the IWT sector especially when more countries are moving to this eco-friendly mode of transport and giving higher priority for IWT.

\section{REFERENCES}

[1] European Commission- Directorate General for Mobility and Transport- Transport research knowledge centre-Modernising inland shipping through advanced information technologiesRiver information services - Multiannual call 2010- May 2010. 
[2] MSP Raju and A. Prem Anandh, Design of Low Wash Catamaran Passenger Ferry for Indian Inland Waterways, International Journal of Civil Engineering and Technology, 9(7), 2018, pp. $1155-1158$.

[3] RIS in EU- European Commission- Directorate General for Energy and Transport- As policy implementation flows from research- Nov.2005; pp. 4.

[4] CCNR Vision 2018 document for sustainable inland navigation- safety and reliability $\mathrm{http}$ //www.inland-navigation.org/observatory/legal-framework/applicable-rules-for-inlandnavigation.

[5] UN Economic Commission for Europe- Inland transport committee- Geneva-Guidelines and recommendations for RIS- Resolution no-57.

[6] A.A. El-Diasty, M. S. El-Sharkawy, Nahla Abu El-Atta and M. M. Nour El-Din, Improving Local-Level Management and Planning Capacity for Waterways Networks in Egypt, International Journal of Civil Engineering and Technology (IJCIET), Volume 6, Issue 2, February (2015), pp. 87-97

[7] Government of Kerala -Shipping and Inland Navigation Department -Kerala Inland Vessels rules -2010 .

[8] European Commission- Directorate General for Transport - Panteia Research to ProgressContribution to impact assessment of measures for reducing emissions of inland navigation: June 10, 2013; pp. 2. 